\section{Infants listen for more phonetic detail in speech perception than in word-learning tasks}

\section{Christine L. Stager \& Janet F. Werker}

Department of Psychology, University of British Columbia, 2136 West Mall, Vancouver, British Columbia V6T 1Z4, Canada

Infants aged 4-6 months discriminate the fine phonetic differences that distinguish syllables in both their native and unfamiliar languages $^{1-3}$, but by 10-12 months their perceptual sensitivities are reorganized so that they discriminate only the phonetic variations that are used to distinguish meaning in their native language $^{12}$. It would seem, then, that infants apply their well honed phonetic sensitivities as they advance and begin to associate words with objects, but the question of how speech perception sensitivities are used in early word learning has not yet been answered. Here we use a recently developed technique to show that when they are required to pair words with objects, infants of 14 months fail to use the fine phonetic detail they detect in syllable discrimination tasks. In contrast, infants of 8 months - who are not yet readily learning words - successfully discriminate phonetic detail in the same task in which infants aged 14 months fail. Taken together, these results suggest a second reorganization in infants's use of phonetic detail as they move from listening to syllables to learning words.

To examine the phonetic information used by infants in early word learning, we used a recently developed procedure in which infants are first taught word-object pairings, and then tested on their ability to detect a change in either the word, the object, or both the word and object (J.F.W., L. B. Cohen, V. L. Lloyd, M. Casasola and C.L.S., manuscript in preparation). Using this procedure, it has been shown that when two-word-object pairings are used, infants aged 14 months, but not younger, are able to associate the dissimilar-sounding nonsense words with their referents (for example, infants learn object A is called "lif" and object B is called "neem") (J.F.W. et al., manuscript in preparation). This may be because younger infants can only easily learn and understand new words under conditions that provide rich contextual support ${ }^{4,5}$.

To determine whether infants can use their speech-perception skills when first learning word-object associations, we tested 14month-old infants in this procedure using phonetically similar nonsense words. If infants are using their fine phonetic discrimination skills, we would expect them to have no trouble learning two words that sound similar. In the first experiment, infants were presented with label A paired with object A and label B paired with object B (or vice versa, with order counterbalanced across infants). The two nonsense labels, "bih" and "dih", were phonetically similar differing in only the place of articulation of the initial consonant. The two brightly coloured moving objects were created from modelling clay and matched for overall visual salience. The visual objects were presented on a monitor situated directly in front of the infant and the words were presented over a loudspeaker located below the monitor. During the habituation phase, the two wordobject pairs were repeated in random order until the infant became familiarized to the pairings, as indicated by a criterial decline in looking time. Following habituation, two test trials were presented: one trial involved the same word-object combination ('same' trial) and the other involved the same words and objects, but a switch in the word-object pairing ('switch' trial) (Fig. 1).

We tested sixty-four 14-month-old infants (mean age, 14 months 16 days; range, 14 months 1 day to 14 months 29 days). Successful discrimination of the fine phonetic detail distinguishing these two nonsense words would be revealed by infants noticing the switch in pairing and looking longer to the 'switch' trial than to the 'same' trial. A $t$-test revealed no significant difference in looking time $[t(63)=0.916 ; \mathrm{NS}]\left(M_{\text {same }}=6.125 \mathrm{~s}, \mathrm{SD}=3.278 ; M_{\text {switch }}=\right.$ $6.584 \mathrm{~s}, \mathrm{SD}=3.775$ ) (Fig. 2). Infants taught these minimally different words did not appear to notice the switch in wordobject pairing.

In the second experiment, we investigated why infants fail to learn phonetically similar words. We tested infants on an even easier, single-word-object association task. We have shown that when the task is simplified to a single-word-object pairing, even much younger infants ( 8 months) can perform successfully (J.F.W. et al., manuscript in preparation). This may be because the single-wordobject association task is passable in two ways: infants who are readily able to link a word with an object may pass the task as a word-learning task, whereas infants who are not yet pairing the word and the object together can pass the task as a simple sounddiscrimination task.

We tested sixteen 14-month-old infants (mean age, 14 months 12 days; range, 14 months 3 days to 14 months 30 days) and sixteen 8 month-old infants (mean age, 8 months 18 days; range, 8 months 4 days to 9 months 2 days). The same words and visual stimuli were used as in experiment 1 , but infants were presented with only a single pair (for example, they were taught object A called "bih" and then tested with object A still called "bih" as the 'same' trial, and object A called "dih" as the 'switch' trial) (Fig. 1). When tested with these minimally different words even in this simplified procedure, infants of 14 months did not notice the switch in the word-object pairing. There was no significant difference in looking time to the 'same' trial as compared to the 'switch' trial $[t(15)=0.242$; NS] $\left(M_{\text {same }}=7.120 \mathrm{~s}, \quad \mathrm{SD}=2.979 ; \quad M_{\text {switch }}=6.888 \mathrm{~s}, \quad \mathrm{SD}=2.965\right)$. However, infants of 8 months did notice the switch in word, and looked significantly longer during the 'switch' trial than during the 'same' trial $[t(15)=2.225, P<0.05]\left(M_{\text {same }}=6.350 \mathrm{~s}, \mathrm{SD}=\right.$ $3.021 ; M_{\text {switch }}=8.198 \mathrm{~s}, \mathrm{SD}=2.592$ ) (Fig. 2). These results indicate that the task is different for infants of 8 and 14 months of age: they suggest that for infants of 14 months, the single word-object association task does involve word learning, and that under these circumstances, the difference between "bih" and "dih" is not noticed. For infants of 8 months, this may be a simple sounddiscrimination task, and the difference between "bih" and "dih" is easily detected.

To ensure that infants of 14 months are able to perform successfully

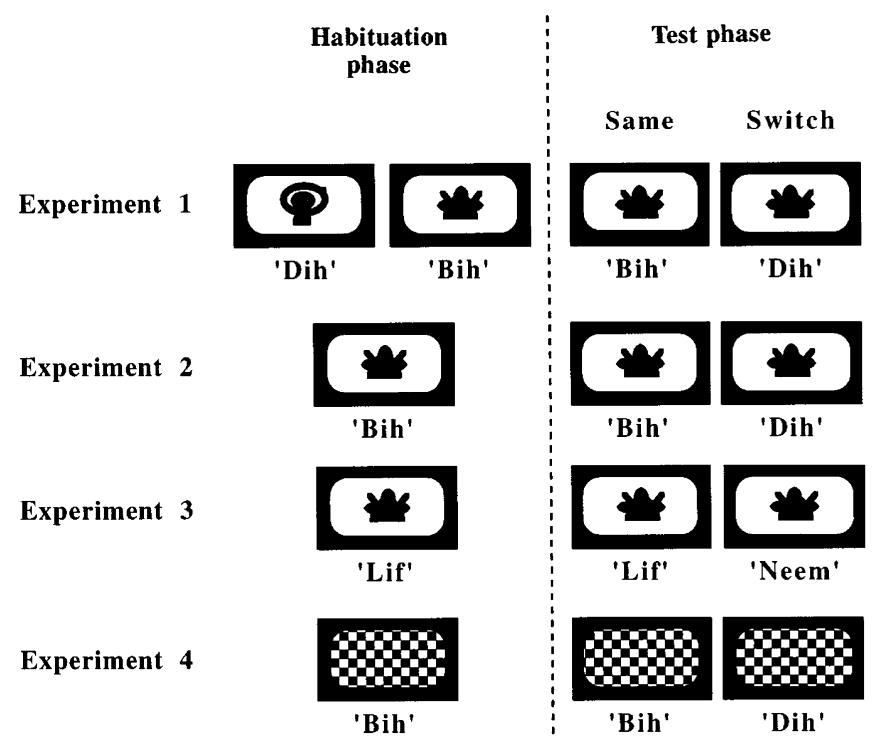

Figure 1 Diagrammatic representations of experiments 1-4. 
in this single word-object association task, we did a control experiment using the phonetically dissimilar words that have previously been used in the two-word-object association task (J.F.W. et al., in preparation) and using the visual objects we had chosen for this series of experiments. The procedure we used in experiment 3 was identical to that used in experiment 2, except that we used the phonetically dissimilar stimuli "lif" and "neem" as the object labels (Fig. 1). Sixteen 14-month-old infants (mean age, 14 months 6 days; range, 13 months 25 days to 14 months 29 days) completed this experiment. Infants clearly discriminated between the two labels $[t(15)=5.412, P<0.0001] \quad\left(M_{\text {same }}=7.984 \mathrm{~s}\right.$, $\left.\mathrm{SD}=2.769 ; M_{\text {switch }}=12.428 \mathrm{~s}, \mathrm{SD}=2.294\right)$ (Fig. 2). This result confirms that the difficulty experienced by 14 -month-old infants in experiment 2 was due to the phonetic similarity of the labels used and not to other task factors.

The results from experiments 1-3 indicate that there is a difference in the phonetic detail used in word-learning versus speech-perception tasks, but the possibility also exists that infants of 14 months can simply no longer make the fine phonetic discriminations they demonstrated when younger. To eliminate this possibility, we did one further experiment with infants aged 14 months. We made the task one of simple speech discrimination by pairing the presentation of the words with a visual display that is unlikely to foster naming. We paired the word with the presentation of a checkerboard pattern on the TV monitor. There is considerable evidence that an unbounded stationary display like a checkerboard is unlikely to be perceived as an object by an infant ${ }^{6}$, and is thus less likely to be labelled ${ }^{7}$. All other aspects of the procedure were identical to those used in the previous experiment.

In this final study (experiment 4), sixteen 14-month-old infants (mean age, 14 months 20 days; range, 14 months 13 days to 14 months 28 days) were shown a checkerboard pattern paired with either the syllable "bih" or the syllable "dih" (Fig. 1). Under these testing conditions, infants of 14 months showed robust evidence of discriminating "bih" from "dih" $[t(15)=3.691, P<0.05]$ $\left(M_{\text {same }}=5.005 \mathrm{~s}, \mathrm{SD}=3.491 ; M_{\text {switch }}=7.116 \mathrm{~s}, \mathrm{SD}=2.959\right)$ (Fig. 2 ). This finding eliminates the possibility that infants of 14 months can no longer make fine phonetic discriminations. Instead, it presents compelling evidence that it is only when infants are attempting to learn the meaning of words that they fail to attend to the fine phonetic information.

Taken together, the results of these four experiments provide convincing evidence that infants use different information in wordlearning than in speech-perception tasks. When listening for mean-

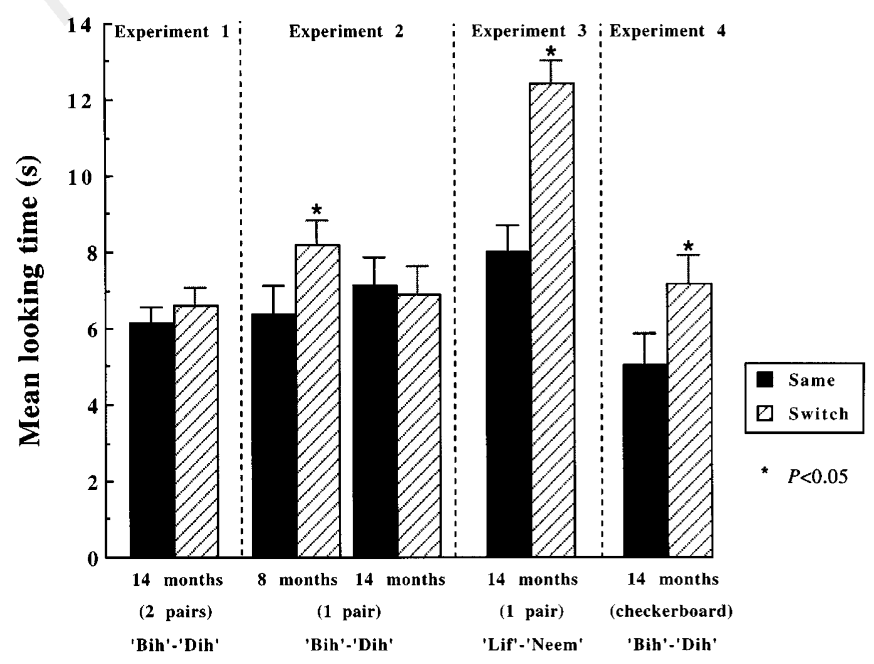

Figure 2 Results showing the conditions under which infants show significant recovery on the 'switch' trials. Graphs show mean looking times on the 'same' and 'switch' trials, with standard error bars. ing (experiments 1 and 2), infants of 14 months fail to detect the same phonetic detail that they can easily detect in a simple syllable discrimination task (experiment 4 ). Infants of 8 months may be able to pass the single-word-object association task used in experiment 2 because they are not mapping the sound onto meaning.

Inattention to phonetic detail may be beneficial to the child who is at the cusp of word learning. The task of linking words with objects is computationally more demanding than just listening to words as sounds. Thus, to be successful at this task, it may be necessary to limit the amount of detail, just as it is in other aspects of perceptual $^{8}$ and linguistic ${ }^{9}$ development. At an older age, when word learning is no longer difficult, we would expect fine phonetic detail again to be accessed. By 3 years of age, English-learning children can distinguish most similar sounding words ${ }^{10}$. But at 14 months, limited phonetic information is all that is needed to avoid confusing a new word with the few words in the infants' lexicon ${ }^{11}$.

The underlying pattern suggested by these results may be considered as another example of functional reorganization ${ }^{3}$. The decrease in the amount of detail used by infants as they move from speech perception to word learning is analogous to the earlier decline in infants' ability to discriminate non-native phones ${ }^{12}$. In both cases, a decline rather than an increase in performance is evidence of a developmental progression. We believe that this type of functional reorganization is a necessary and ubiquitous aspect of development. Being aware of and understanding functional reorganizations may help us to resolve some of the puzzles of early development.

\section{Methods}

The infant sat on the parent's lap facing the video screen. To prevent any biasing influence from seeing or hearing the display, parents wore a hat with a visor and a cloth blind and listened to female vocal music over headphones. The experimenter initiated trials when the infant visually fixated on a flashing red light in the centre of the monitor. The experimenter was seated in an adjoining control room, and recorded each infant's looking time on-line on a closed circuit TV system. The experimenter was unaware of the auditory stimulus being presented to the infant. Reliability coding conducted on $25 \%$ of the infants yielded reliability coefficients for each group of $>0.90$. Across all four experiments, infants were excluded if they started to fuss during the experiment ( $n=32$ ), if they were not visible for on-line recording or filming $(n=13)$, if parents interfered during testing $(n=9)$, if the infant did not habituate $(n=1)$, if there was equipment failure $(n=2)$, or if infants did not look during one of the test trials $(n=1)$.

Received 16 January; accepted 2 May 1997.

1. Kuhl, P. K. in Handbook of Infant Speech Perception Vol. 2 (eds Salapatek, P. \& Cohen, L.) 275-382 (Academic, New York, 1987)

2. Jusczyk, P. W. in Handbook of Perception and Cognition, 11: Speech, Language, and Communication (eds Miller, J. L. \& Eimas, P. D.) 263-301 (Academic, Orlando, Florida, 1995).

3. Werker, J. in Invitation to Cognitive Science: Language (eds Gleitman, L. R. \& Liberman, M.) 87-106 (MIT Press, Cambridge, MA, 1995).

4. Benedict, H. Early lexical development: Comprehension and production. J. Child Lang. 6, 183-200 (1979).

5. Oviatt, S. L. The emerging ability to comprehend language: An experimental approach. Child Dev. 51, 97-106 (1980).

6. Spelke, E. S., Vishton, P. \& Van Hofsten, C. in The Cognitive Neurosciences (ed. Gazzaniga, M.) 165179 (MIT Press, Cambridge, MA, 1994).

7. Woodward, A. in Twenty-fourth Annual Child Language Research Forum (ed. Clark, E.) 35-45 (Cambridge University Press, Cambridge, MA, 1993).

8. Turkewitz, G. \& Kenny, P. A. Limitations on input as a basis for neural organization and perceptual development: A preliminary theoretical statement. Dev. Psychobiol. 15, 357-368 (1982).

9. Newport, E. Maturational constraints on language learning. Cog. Sci. 14, 11-28 (1990).

10. Barton, D. The Development of Communication (eds Waterson, N. \& Snow, C.) 255-261 (Wiley, New York, 1978).

11. Charles-Luce, J. \& Luce, P. A. An examination of similarity neighborhoods in young children's receptive vocabularies. J. Child Lang. 22, 727-735 (1995).

12. Werker, J. F. \& Tees, R. C. Cross-language speech perception: Evidence for perceptual reorganization during the first year of life. Infant Behav. Dev. 7, 49-63 (1984).

Acknowledgements. We thank J. A. R. Carr and R. C. Tees for comments on earlier drafts, and $M$. Bhatnager for her help in testing infants. We also thank the parents and infants who participated in the study. This research was supported by a Natural Sciences and Engineering Research Council of Canada Grant to J.F.W. and a NSERC Doctoral Scholarship to C.L.S.

Correspondence and requests for materials should be addressed to C.L.S. (e-mail: cstager@unixg.ubc.ca) or J.F.W. (e-mail: jwerker@cortex.psych.ubc.ca). 